\title{
Deficiencia de biotinidasa.
}

Revisión

Jazminia A. Moreno-Arango, Lixandra Texidor-Llopiz.

Laboratorio de Errores Innatos del Metabolismo. Centro Nacional de Genética Médica. La Habana, Cuba.

\section{RESUMEN.}

Objetivos. Brindar información acerca de la deficiencia de la enzima biotinidasa.

Fuentes. Libros de texto relacionados con el tema y artículos actualizados de revistas.

Resultados. Los errores innatos del metabolismo se caracterizan por alteraciones en la síntesis ó en el funcionamiento de algunas enzimas que participan en diferentes procesos metabólicos en el organismo, uno de estos defectos es la deficiencia de biotinidasa. La biotinidasa es la enzima responsable del reciclaje de la biotina, a partir de la biocitina o de pequeños péptidos biotinilados y de la liberación de la vitamina unida a las proteínas de la dieta. La biotina es una vitamina perteneciente al complejo B que en humanos actúa como grupo prostético de cuatro carboxilasas esenciales en procesos metabólicos, tales como: gluconeogénesis, síntesis de ácidos grasos y catabolismo de aminoácidos. La deficiencia de biotinidasa es un desorden de herencia autosómica recesiva del metabolismo de la biotina, causado por ausencia ó deficiencia de la enzima. Estudios genéticos han determinado que el gen que codifica para la biotinidasa se localiza en el cromosoma 3p25. Los pacientes afectados con la enfermedad pueden presentar crisis convulsivas, hipotonía, ataxia, dermatitis, alopecia y problemas neurológicos. El diagnóstico temprano y la terapia con dosis substitutivas de biotina previene la aparición de las manifestaciones clínicas y las alteraciones bioquímicas. Conclusiones. La deficiencia de biotinidasa es un desórden de herencia autosómica recesiva causado por ausencia ó deficiencia de la enzima y los síntomas no son más que el resultado de la incapacidad por parte de los pacientes de reutilizar la biotina.

(Rev Biomed 2005; 16:185-191)

Palabras clave: deficiencia de biotinidasa, biotina, error innato del metabolismo.

\section{SUMMARY.}

\section{Biotinidase deficiency.}

Objectives. To offer information about biotinidase deficiency.

Sources. Text books and articles.

Results. Inborn errors of metabolism are characterized by alterations in the synthesis or working of some enzymes that participate in different processes

Solicitud de sobretiros: Lic. Jazminia A. Moreno-Arango, Centro Nacional de Genética Médica, Centro colaborador de la OMS para el desarrollo de enfoques genéticos en la promoción de salud. Calle 146 No. 3102 esq. ave 31. Marianao C.P. 11600. La Habana, Cuba. Correo electrónico: jazminia@giron.sld.cu 
in the organism, one of these defects is biotinidase deficiency. Biotinidase is the enzyme responsible for cleaving biotin from biocytin or from short biotnyl peptides and for liberating the vitamin from dietary protein-bound sources. In humans, biotin is a B complex vitamin that acts as the prosthetic group of four carboxilases, essential in metabolic processes such as: gluconeogenesis, fatty acid synthesis, and amino acid catabolism. Biotinidase deficiency is an autosomal recessive disorder of biotin metabolism, caused by the absence or deficiency of the enzyme, and genetic studies have determined the human gene is located in chromosome 3p25. Patients affected with the disease show convulsive crises, hypotonia, ataxia, skin rash, alopecia, and neurological problems, but early diagnosis and therapy with substitutive doses of biotin prevent clinical manifestations and biochemical alterations.

Conclusions. Biotinidase deficiency is an autosomal recessive disorder caused by the absence or deficiency of the enzyme and the symptoms result from the inability to reutilize biotin.

\section{(Rev Biomed 2005; 16:185-191)}

Key words: biotinidase deficiency, biotin, inborn errors of metabolism.

\section{INTRODUCCIÓN.}

Los errores innatos del metabolismo son enfermedades monogénicas de herencia autosómica recesiva en su mayoría. Dentro de ellos tenemos la deficiencia o ausencia de la enzima biotinidasa, lo cual provoca alteraciones en el metabolismo de la biotina. Este desorden aparece entre el tercer y sexto mes de vida, pero puede manifestarse tan temprano como en la primera semana de nacimiento el niño y tan tarde como a los 10 años de edad (1). Los síntomas suelen ser deterioro neurológico con retardo, convulsiones ataxia, lesiones de la piel, alopecia y acidosis metabólica entre otros. Se ha establecido para su tratamiento la biotina, vitamina termoestable perteneciente al complejo $\mathrm{B}$, reconocida desde el año 1936 que se conoce también como vitamina $\mathrm{H} \mathrm{o}$ vitamina B8 y coenzima, se absorbe por el intestino delgado para luego distribuirse a todos los tejidos del organismo (2). Su administración es de por vida porque a pesar de que no cura la enfermedad, asegura una rápida mejoría clínica, evita la reaparición de las manifestaciones clínicas y no ocasiona daños al paciente. La incidencia de la enfermedad a nivel mundial se estima que es 1:110,000 para la deficiencia total y 1:60,000 para deficiencia parcial (3).

\section{BREVE RESEÑA HISTÓRICA.}

En la década de los cincuenta, Thoma y Peterson determinaron la presencia de una enzima en el hígado del cerdo que era capaz de liberar la biotina que formaba parte de los péptidos digestivos (4). Casi simultáneamente Wright y colaboradores describieron una enzima en el plasma humano que hidrolizaba la biocitina (producto de la degradación proteolítica de las carboxilasas). Esta enzima presente en el hígado del cerdo y en plasma humano se conoce como biotina amida amidohidrolasa $(5,6)$ y comúnmente se le denomina biotinidasa. Aunque se conocía desde mediados del siglo, es hasta la década de los ochenta que Wolf y colaboradores muestran que su deficiencia es el defecto bioquímico en pacientes con la forma de presentación tardía o juvenil de deficiencia múltiple de carboxilasa (3).

\section{LA ENZIMA BIOTINIDASA. CARAC- TERÍSTICAS.}

La biotinidasa es una glicoproteína monomérica con un peso molecular de 76 a $77 \mathrm{kDa}$ (7) y tiene 9 isoformas. Está presente en diferentes especies y tejidos y ha sido detectada en microorganismos, anfibios, aves y mamíferos $(8,9)$. Posee una actividad enzimática elevada en suero, hígado, riñón y glándula suprarrenal. Además está presente en el jugo pancreático, en la orina humana y en las células secretoras $(8,10-12)$. Tiene las siguientes funciones: actividad hidrolítica, biotiniltransferasa, lipoamidasa y como proteína transportadora de biotina (13). De todas, la más estudiada ha sido la hidrolítica, capaz de hidrolizar d-biotinilamidas y ésteres $(8,9)$ pero incapaz de hidrolizar el enlace formado entre las holocarboxilasas y la biotina $(14,15)$.

\section{Revista Biomédica}


Deficiencia de Biotinidasa.

METABOLISMO DE LA BIOTINA.

La deficiencia de biotinidasa es una irregularidad metabólica reconocida como el defecto primario en la deficiencia múltiple de cuatro carboxilasas dependientes de biotina. La holocarboxilasa es la encargada de catalizar la unión de la biotina a las carboxilasas mediante un enlace amida con los grupos epsilon-amino de residuos específicos de lisina de las apocarboxilasas (16), de esta forma se convierten en proteínas enzimáticamente activas y pueden desempeñar sus funciones en los diferentes procesos metabólicos en que están involucradas, tales como el catabolismo de aminoácidos, la lipogénesis y la gluconeogénesis, además de transferir dióxido de carbono.

La acetil CoA carboxilasa es citosólica, su función es catalizar la conversión de acetil $\mathrm{CoA}$ a malonil $\mathrm{CoA}$ lo que constituye la etapa limitante de la biosíntesis de los ácidos grasos (17).

El resto de las carboxilasas son mitocondriales $\mathrm{y}$ entre ellas tenemos a:

La piruvato carboxilasa, que además de participar en la gluconeogénesis también interviene en el mantenimiento de los niveles de intermediarios del ciclo del ácido cítrico. Dicho metabolito al acumularse sale de las mitocondrias, en el citoplasma es un precursor para los procesos de síntesis de ácidos grasos y la esteroidogénesis (18).

La propionil CoA carboxilasa tiene como función la carboxilación del propionil $\mathrm{CoA}$, convirtiéndolo en metil malonil CoA. Luego pasa a succinil CoA y forma parte del ciclo de los ácidos tricarboxílicos.

Por último, la metil crotonil CoA carboxilasa, que interviene en el paso de $\beta$ metilcrotonil CoA a metil glutaconil CoA en el catabolismo de la Leucina (1).

La biotinidasa desempeña una función importante en el reciclaje de la biocitina, producto conocido como el complejo biotina-lisina que se obtiene de la degradación de las carboxilasas. Debido a su actividad hidrolítica este complejo se divide, quedando la biotina libre para ser utilizada por las carboxilasas, además de encargarse de separar la biotina unida a las proteínas de la dieta llevándolas a una forma biológicamente viable. De existir una deficiencia en su funcionamiento, todo este proceso se ve afectado provocando trastornos en la formación de las holocarboxilsas.

\section{Cuadro 1}

Metabolismo de la Biotina.

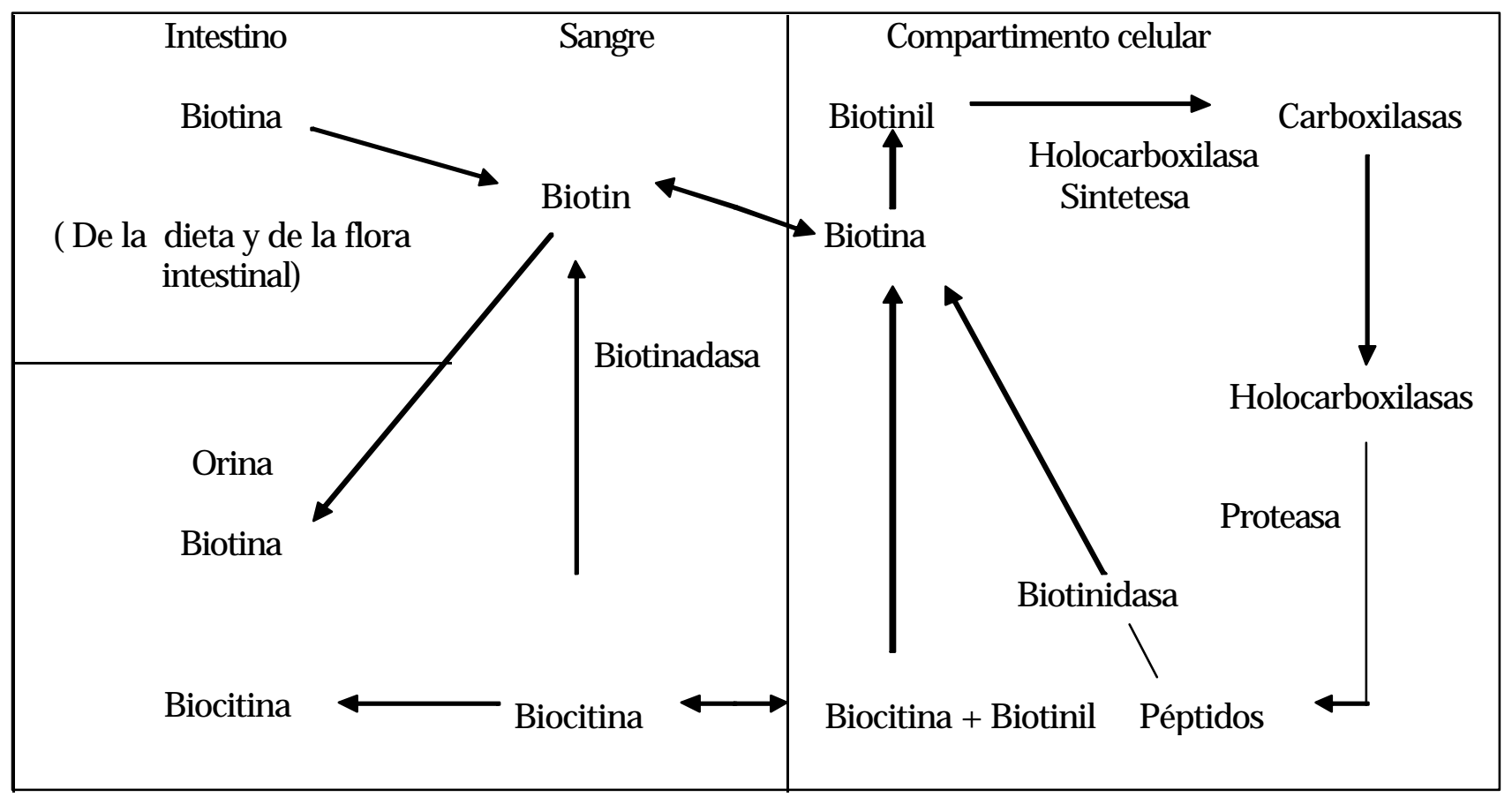




\section{BASES MOLECULARES DE LA ENFERMEDAD.}

El gen que codifica para esta enzima se localiza en el cromosoma 3p25(19) está organizado dentro de cuatro exones con tallas de $79,265,150$ y 1502 bp, respectivamente. Se han identificado más de 100 mutaciones en niños con deficiencia de biotinidasa profunda pero la más comunes son: G98:d7i3 y 1612C? T(538? C9) aunque también se han reportado las mutaciones 1368A? C (456Q? H) y la doble mutación 511G? A:1330G? C(171A? T:444D? H).

La deficiencia parcial usualmente ocurre cuando un individuo tiene un alelo que resulta en pérdida total de la actividad en combinación con un alelo que tiene la mutación 1330G? C (444D? H(20,21,22).

\section{RELACIÓN FENOTIPO-GENOTIPO.}

Ciertos genotipos están relacionados con la deficiencia parcial y otros con la deficiencia profunda de biotinidasa. Se conoce que muchas mutaciones causan la pérdida completa de la actividad de la enzima como por ejemplo, individuos que son heterocigóticos y presentan la mutación 444D? Hy una mutación que resulta una deficiencia profunda se espera que tengan alrededor de un $20-25 \%$ de la actividad normal de la enzima en suero (21).

También la mutación 444D? Hen configuración cis con la mutación 141A? T resulta en un alelo que causa deficiencia de biotinidasa profunda, por lo tanto un individuo con un alelo teniendo estas dos mutaciones en configuración cis combinada con otro alelo con una mutación para deficiencia de biotinidasa profunda tendrá deficiencia profunda de la actividad de la enzima (22).

Los individuos que son homocigóticos y tienen dos alelos para la mutación 444D? H tienen de un $45-50 \%$ de la actividad normal de biotinidasa en suero.

\section{MANIFESTACIONES CLÍNICAS.}

Los síntomas de la enfermedad se deben a una acidosis (estado metabólico en el que existen cantidades anormales de cuerpos cetónicos) en la sangre y en diferentes tejidos del organismo.

Los síntomas clínicos usualmente aparecen durante la primera semana de vida y los 10 años, aunque se han reportado casos que han desarrollado los síntomas en la adolescencia $(23,24)$ y otros asintomáticos que han sido diagnosticados porque sus hijos afectados se han identificado en el tamizaje neonatal. Por ejemplo, un hombre caucasiano de 32 años quien nunca tuvo manifestaciones de este desorden y mostró no tener anormalidades físicas ó neurológicas pero que además no tomaba suplementos vitamínicos ni llevaba una dieta rica en alimentos que usualmente contienen biotina como por ejemplo el huevo. Y otro caso reportado es el de una mujer caucasiana de 36 años detectada a través de su hija de 15 años con deficiencia de biotinidasa profunda pero también sin síntomasa clínicos a no ser rash cutáneo en los primeros meses de vida (25). Este trastorno en el cual el organismo no puede procesar de manera correcta la biotina exógena y endógena (19) se caracteriza principalmente por hipotonía, rash cutáneo, ataxia, conjuntivitis, infecciones de hongos, pérdida de la audición y dentro de los problemas respiratorios podemos citar hiperventilación, apnea y estridor laringeal. Además en la forma más temprana se va a presentar con una excreción en la orina de los ácidos 3- hidroxivalérico y 3- hidroxipropiónico (26). Las alteraciones bioquímicas más frecuentes son acidosis cetoláctica y aciduria orgánica, conjuntamente con un incremento en la concentración de amoníaco (27), lo cual puede ocasionar daños en importantes órganos como el cerebro, oído interno y ojos.

\section{CLASIFICACIÓN.}

Se ha establecido su clasificación atendiendo al porcentaje de la actividad enzimática. Se considera deficiencia total cuando el individuo presenta un $10 \%$ de la actividad hidrolítica normal, la incidencia ha sido estimada en 1:110,000 y deficiencia parcial cuando los niveles se encuentran entre el 10\%-30\% con una incidencia de 1: 60,000 (28).

\section{Revista Biomédica}


Deficiencia de Biotinidasa.

\section{DIAGNÓSTICO.}

El tamizaje neonatal es muy útil en este sentido ya que se realiza con el objetivo de detectar trastornos que no se evidencian inmediatamente después del parto. Estos trastornos pueden ser genéticos, metabólicos sanguíneos u hormonales. Para analizar la sangre del bebé se realiza un pinchazo en el talón del mismo y las gotas de sangre se recogen en un frasquito o en un papel especial para luego realizarle el análisis. No son pocas las enfermedades que pueden ser detectadas por esta vía, entre ellas se encuentran: Fenilquetonuria (PKU), Hipotiroidismo congénito, Galactosemia, Anemia drepanocítica, Hiperplasia suprarrenal congénita y Deficiencia de biotinidasa donde el diagnóstico y tratamiento tempranos son fundamentales para evitar el daño irreversible del sistema nervioso central y la muerte provocada por la acidosis metabólica. Además la existencia de métodos de diagnóstico simples y poco costosos y la positiva relación costo-beneficio para la sociedad hacen que la deficiencia de biotinidasa cumpla con los criterios de inclusión en los programas de tamizaje neonatal. De ahí que países como Estados Unidos, Bélgica, España, Argentina, Italia, Turquía, Suiza y Brasil hallan incursionado en el tamizaje de esta enfermedad (2).

Para el diagnóstico de deficiencia de biotinidasa varios tipos de ensayos y sustratos han sido utilizados entre ellos se incluyen mediciones enzimáticas, radiométricas, colorimétricas y fluorimétricas. El método más utilizado es el que emplea Biotinil-Ácidop- aminobenzoico como sustrato, el cual se utiliza tanto para el tamizaje neonatal de la enfermedad como para el diagnóstico de niños con síntomas clínicos. Es un método colorimétrico que se basa en la determinación de los niveles de ácido p- aminobenzoico liberados producto de la acción hidrolítica de la enzima (29, 30) y empleando modificaciones de este método ha podido determinarse cualitativa y cuantitativamente la actividad de la enzima en cultivos celulares, sueros y sangre seca colectada en papel de filtro. Otro método muy empleado es el que se basa en la medición fluorimétrica de 6-aminoquinolina, obtenido de la hidrólisis del sustrato biotinil-6-aminoquinolina (31, 32). También aunque se encuentra en fase de investigaciones se ha determinado que el diagnóstico molecular podría ser de gran utilidad ya con las técnicas de secuenciación de ADN se detectan el $100 \%$ de las mutaciones del gen y con PCR las mutaciones G98:d7i3, Q456? H, R538? C, D444? Hy D444? H:171A? T(33).

\section{TRATAMIENTO.}

La biotina es un factor esencial en la nutrición humana. Su deficiencia es muy rara, debido al gran número de alimentos donde está presente y las pequeñas cantidades de biotina que son requeridas por el organismo, si se compara con otros nutrientes esenciales. Los mamíferos son incapaces de sintetizar esta vitamina y necesitan obtenerla de la dieta del reciclaje de la biotina endógena (2). Se ha establecido que en pacientes con defecto total de la enzima y diagnóstico neonatal la suplementación oral con 10$20 \mathrm{mg}$ diarios de biotina revierte o previene el cuadro clínico y bioquímico siendo necesario mantenerlo durante toda la vida. Cuando la deficiencia es parcial, se requiere de una dosis de 2,5-5 mg por semana (34).

\section{RELACION DE LA DEFICIENCIA DE BIOTINIDASA CON OTRAS ENFERMEDADES.}

Todos los individuos con deficiencia de biotinidasa no desarrollan los síntomas pero si existen varios casos que no han sido procesados mediante el tamizaje neonatal, donde se ha demostrado que tienen la enfermedad e incluso asociada a otra. Tal es así que Grunewald y col en el 2004 reportaron cinco pacientes los cuales en el período de 4 a 5 meses presentaron leucoencefalopatía y después del tratamiento se observó la mejoría con biotina a pesar de que quedaron con secuelas neurológicas. Y Puertas $y$ col en el 2004 estudiaron a un niño de 12 años con pérdida de la visión en ambos ojos, asma, dermatitis y alopecia, al cual se le diagnosticó neuropatía retrobulbar óptica y también resultó ser deficiente de biotinidasa, rápidamente se le comenzó a administrar biotina y quedó demostrado que esta terapia es satisfactoria en la neuropatía óptica $(35,36)$.

Vol. 16/No. 3/Julio-Septiembre, 2005 


\section{JA Moreno-Arango, L Texidor-Llopiz.}

\section{REFERENCIAS.}

1.- Scriver CH R, Beaudet AL, Sly WS, Valle D. Disorders of biotin metabolism. En: Scriver CH, Beaudet A, Sly W, Valle $\mathrm{D}$, editores. The metabolic and molecular bases of inherited disease. 7a ed. New York: McGraw-Hill Publishing Co.; 1995 Vol II. p. 3151-72.

2.- González Reyes E, Marrero González N. Deficiencia de biotinidasa. Bioquimia 2002; 27:80-86.

3.- Wolf B, Grier RE, Parker WD, Goodman SI, Allen RJ. Deficient Biotinidase activity in late-onset multiple carboxylase deficiency. N Engl J Med 1983; 308: 161.

4.- Thoma R W, Peterson W H. The enzymatic degradation of soluble bound biotin J Biol Chem 1954: 210-410.

5.- Harper PS: Practical genetic counseling. $5^{\text {th }}$ ed. HPS eds. Butterworth Heinemann. Boston:1999.

6.- Thompson MW, Mclnnes RR, Willard HF. Diagnóstico prenatal. En: Thompson MW, Mclnnes RR, Willard HF. Genética en medicina. $4^{\text {ta }}$ ed. Masson: 1996. p. 395-409.

7.- Hart PS, Hymes J,Wolf B. Isoforms of human serum biotinidase. Clin Chim Acta 1991; 197: 257-64.

8.- Pispa J. Animal biotinidase. Ann Med Exp Biol Fenn 1965; 43 (suppl 5): 1-39.

9.- Knappe J, Brunner W, Bederbick KH. Reinigung und eigenschaften der biotinidase aus schweinenieren und Lactobacillus casei. Biochem Z 1963; 338: 599-613.

10.- Wolf B, Heard GS, McVoy JS, Raetz HM. Biotinidase deficiency: The possible role of biotinidase in the processing of dietary protein-bound biotin. J Inher Metab Dis 1984; 7: 121-2.

11.- Heard GS, Grier RE, Weiner DL, McVoy JR, Wolf B. Biotinidase deficiency. Ann NY Acad Sci 1985; 447:252-62.

12.- De Felice C, Hayakawa K, Tanaka T, Terentieva E. Biotinidase activity in the urine of healthy subjects. Nephron 1995;70:115.

13.- Hymes J, Fleischhaver K, Wolf B. Biotinidase in serum and tissues. Methods Enzymol 1997; 279: 422-34.

14.- Craft DV, Goss NH, Chandramouli N, Wood HG. Purification of biotinidase from human plasma and its activity on biotinil peptides. Biochemistry 1985; 24: 2471-6.
15.- Chauhan J. Dakshinamurti K. Purification and characterization of human serum biotinidase. J Biol Chem 1986; 261:4268-75.

16.- Wolf B, Heard GS. Disorders of biotin metabolism. En: Scriver CR, Beaudet Al, Sly WS, Valle D, editores. The metabolic and molecular bases of inherited disease. 6a. ed. New York: McGraw-Hill Publishing Co. 1989. p. 2083-103.

17.- Cardellá RL, Hernández FR. Metabolismo de la glucosa. En: Cheping N, editor. Bioquímica Médica. C. Habana: Ciencias Médicas. $1^{\text {era }}$ ed, 1999. Vol. III. p. 830-47.

18.- Stryer L. Glycolysis. En: Stryer L, editor. Biochemistry. Madrid-Barcelona: Reverte, S.A. $2^{\text {da }}$ ed. 1982. p. 307-27.

19.- Pomponio RJ, Reynolds TR, Cole H, Buck GA, Wolf B. Mutational "Hotspot" in the human biotinidase gene as a cause of biotinidase deficiency. Nat Genet 1995; 11: 96-8.

20.- Wolf B, Jensen K. Seventeen novel mutations that cause profound biotinidase deficiency. Mol Genet Metab 2002; 77:108-11.

21.-Swango KL, Demirkol M ,Huner G, Wolf B .Partial biotinidase deficiency is usually due to the $\mathrm{D} 444 \mathrm{H}$ mutation in the biotinidase gene. Hum Genet 1998; 102: 571-5.

22.- Norrgard KJ, Swango KL, Hymes J, Wolf B. Double mutation[A171T and D444H] is common cause of profound biotinidase deficiency in children ascertained by newborn screening in the United States. Hum Mutat 1998; 11:410.

23.- Rahman S, Standing S, Dalton RN, Pike MG. Late presentation of biotinidase deficiency with acute visual loss and gait disturbance. Dev Med Child Neurol 1997; 39: 830-1.

24.- Wolf B, Pomponio RJ, Norrgard KJ, Lott IT, Baumgartner ER, Suormala T, et al. Delayed-onset profound biotinidase deficiency. J Pediatr 1998; 132: 362-5.

25.- Wolf B, Norrgard K, Pomponio RJ, Shapiro S.Profound biotinidase deficiency in two asymptomatic adults. Am J Med Genet 1997; 73:5-9.

26.- Sweetman L. Two forms of biotin-responsive multiple carboxylase deficiency. J Inherit Metab Dis 1981; 4:53-4.

27.- Wastell HJ, Bartlett K, Dale G, Shein A. Biotinidase deficiency: a survey of 10 cases. Arch Dis Child 1988; 63: $1244-9$.

\section{Revista Biomédica}


28.- Wolf B.Worldwide survey of neonatal screening for biotinidase deficiency. J Inher Metab Dis 1991; 14:923-7.

29.- Wolf B, Grier RE, Allen RJ, Goodman SI, Kien CL. Biotinidase deficiency: the enzymatic defect in late-onset multiple carboxylase deficiency. Clin Chim Acta 1983; 131: 273-81.

30.- Heard GS, McVoy JRS, Wolf B. A Screeing Method for biotinidase deficiency in newborns. Clin Chem 1984; 30: 1257.

31.- Wastell H, Dale G, Bartlett. A Sensitive fluorimetric rate assay for biotinidase using a new derivate of biotin, biotinyl6-aminoquinoline. Anal Biochem 1984; 140: 69-73.

32.- Hymes J, Fleischhaver K, Wolf B. Determination of biotinidase activity with biotinyl -6 aminoquinoline as substrate. Methods Enzymol 1997; 279:442-51.

33.- Dobrowolski SF, Angeletti J, Banas RA. Real time PCR assays to detect common mutations in the biotinidase gene and application of mutational analysis to newborn screening for biotinidase deficiency. Mol Genet Metab 2003; 78:100-7.

34.- Colombo CM, Cornejo EV, Raiman BE. Errores innatos en el metabolismo de los aminoácidos. En: Colombo CM, Cornejo EV, Raiman BE, editores. Errores innatos del metabolismo del niño. 2 da ed. Santiago de Chile: Universitaria. 2003.p.98-99.

35.- Grunewald S, Champion MP, Leonard JV, Morris A. Biotinidase deficiency a treatable leukoencephalopathy. Neuropediatrics 2004; 35:211-6.

36.- Puertas D, Martin C, Duat A. Optic Neuropathy in biotinidase deficiency. Arch Soc Esp Oftalmol 2004; 79:3936. 